\title{
NON-PUERPERAL UTERINE INVERSION ASSOCIATED WITH A PELVIC MASS
}

\author{
Yasmin Sardar ${ }^{1}$, Najla Fasih ${ }^{2}$ \\ ${ }^{1}$ Department of Obstetrics and Gynaecology, The Ottawa Hospital, University of Ottawa, Ontario, Canada, \\ ${ }^{2}$ Department of Radiology, The Ottawa Hospital, University of Ottawa, Ontario, Canada \\ Received: 21 April 2015 / Accepted: 24 April 2015
}

A 63-year-old, postmenopausal, multiparous woman presented with postmenopausal bleeding and a vaginal mass. Pelvic examination revealed a $5 \mathrm{~cm}$ mass protruding from the cervical canal. Abdominal examination was unremarkable. A magnetic resonance imaging (MRI) of the pelvis was requested and endometrial biopsy was obtained.

MRI findings: Sagittal T2W MRI image through the pelvis demonstrates a large, heterogeneous, intermediate signal intensity mass centred on the endometrial cavity, with growth into the cervix [Figure 1]. The tumour is attached to the inverted fundus, with prolapse into the external os as well as vagina. Although there is distention of the vaginal fornices, there is no convincing invasion of the vagina. Post-contrast T1W images (not shown) confirm solid, areas of flame-type enhancement, typical for an inverted uterine sarcoma.

An endometrial biopsy was performed and revealed serous adenocarcinoma of the uterus, suspicious for carcinosarcoma. The patient underwent total abdominal hysterectomy with bilateral salpingo-oophorectomy, omentectomy and tumour debulking. Surgical staging was consistent with Stage IV disease. Histopathology confirmed poorly differentiated heterologous carcinosarcoma (mixed Mullerian mesodermal tumour) with serous epithelial components. Her post-operative recovery was uneventful. In view of her high-grade tumour histology, chemotherapy was recommended.

Carcinosarcoma of the uterus (also known as malignant mixed Mullerian tumour [MMMT]) is a rare and highly aggressive form of uterine cancer. ${ }^{[1]}$ Uterine inversion in a non-pregnant woman is a rare occurrence, with only 150 cases reported from 1887 to $2006{ }^{[2]} 85 \%$ of these cases were related to benign uterine pathologies such as prolapse and submucosal uterine leiomyoma. $15 \%$ of

Correspondence: Dr. Najla Fasih, Department of Radiology, The Ottawa Hospital, University of Ottawa, Ontario, Canada. Email: nfasih@toh.on.ca

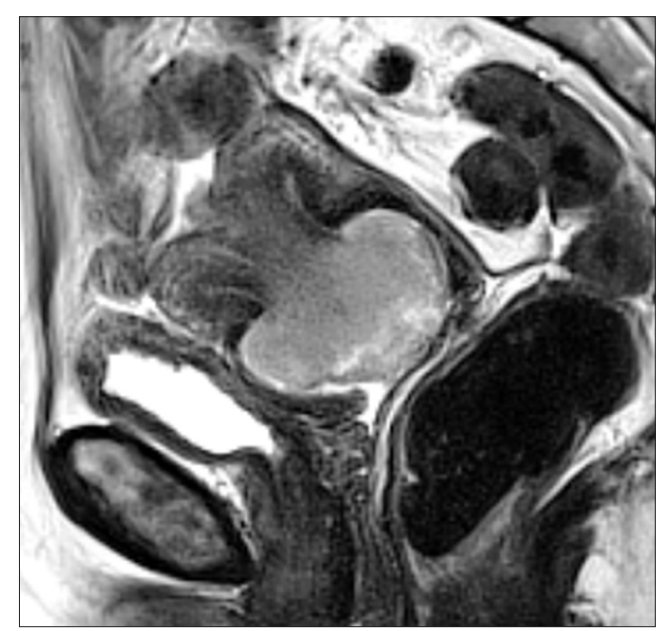

Figure 1: Sagittal T2W MRI image through the pelvis demonstrates a large, heterogeneous, intermediate signal intensity mass centred on the endometrial cavity, with growth into the cervix

cases were secondary to malignant uterine tumours..$^{[3]}$ The exact mechanism of non-puerperal uterine inversion is unknown. However, the condition is typically associated with the presence of a polypoid uterine mass. ${ }^{[2]}$ Possible mechanisms underlying uterine prolapse include (a) sudden extrusion of a tumour from the uterus, (b) thin uterine wall, (c) dilatation of the uterine cervix, (d) large tumour size and (e) thick tumour pedicle. ${ }^{[3]}$

Patients may present with pelvic pain, abnormal uterine bleeding, a pelvic mass or anuria. ${ }^{[4]}$ Unlike puerperal uterine inversion, haemodynamic shock is uncommon. ${ }^{[3]}$ Non-puerperal uterine inversion may be diagnosed clinically, radiologically or at the time of surgery. ${ }^{[5]}$

Clinically, the diagnosis may be suspected if there is a large vaginal mass and difficulty in palpating the cervix. ${ }^{[4]}$ MRI is useful for diagnosing inversion and will often demonstrate a U-shaped uterine cavity with ovaries retracted to the midline. ${ }^{[3]}$ 
Standard, evidence-based treatment includes bilateral salpingo-oophorectomy with pelvic lymph node dissection. Adjuvant radiation therapy, chemotherapy and/or hormonal therapy may be appropriate for some patients, depending on the extent of disease. ${ }^{[2]}$ Resection of the inverted uterus may necessitate an incision in the uterine wall in rare cases of complete uterine inversion with a tight constriction ring.

\section{Conclusion}

Non-puerperal uterine inversion occurs infrequently and is usually associated with benign pathology. However, in postmenopausal women, a high index of suspicion for uterine malignancy, particularly sarcoma, should be maintained.

\section{Conflict of Interest}

The authors declare that they have no conflict of interest.

\section{References}

1. Kernochan LE, Garcia RL. Carcinosarcomas (malignant mixed mullerian tumour) of the uterus: Advances in elucidation of biologic and clinical characteristics. J Natl Compr Canc Netw 2009;7:550-6.

2. Lupovitch A, England ER, Chen R. Non-puerperal uterine inversion in association with uterine sarcoma: Case report in a 26-year-old and review of the literature. Gynecol Oncol 2005;97:938-41.

3. Takano K, Ichikawa Y, Tsunoda H, et al. Uterine inversion caused by uterine sarcoma: A case report. Jpn J Clin Oncol 2001;31:39-42.

4. Moodley M, Moodley J. Non-puerperal uterine inversion in association with uterine sarcoma: Clinical management. Int J Gynaecol Cancer 2003;13:244-5.

5. Buyukkurt S, Vardar MA, Zeren $\mathrm{H}$, et al. Non-puerperal inversion of the uterus caused by leiomyosarcoma: A case rep and clinical management. J Obstet Gynaecol Res 2007;33:402-6. 